\title{
Analysis of gene expression profiles of non-small cell lung cancer at different stages reveals significantly altered biological functions and candidate genes
}

\author{
JIN WANG $^{1 *}$, JIANXIANG SONG ${ }^{1 *}$, ZHENGYA GAO $^{1}$, XUDONG HUO $^{1}$, YAJUN ZHANG $^{1}$, \\ WENCAI WANG $^{1}$, JIANWEI QI ${ }^{1}$ and SHIYING ZHENG ${ }^{2}$ \\ ${ }^{1}$ Department of Cardiothoracic Surgery, Yancheng Hospital, Medical School of Southeast University, \\ The Third People's Hospital, Yancheng, Jiangsu 224001; ${ }^{2}$ Department of Cardiothoracic Surgery, \\ The First Affiliated Hospital of Suzhou University, Suzhou, Jiangsu 215006, P.R. China
}

Received June 14, 2016; Accepted November 10, 2016

DOI: $10.3892 /$ or.2017.5380

\begin{abstract}
We attempt to dissect the pathology of non-small cell lung cancer (NSCLC) patients at different stages and discover the novel candidate genes. Microarray data (GSE21933) were retrieved from the Gene Expression Omnibus database. The differential expression profiles of lung tumor tissues during different stages were analyzed. The significantly altered functions and pathways were assessed and the key nodes in a protein-protein interaction (PPI) network were screened out. Then, the coexpression gene pairs and tumor-related genes were assessed. RT-PCR analysis was performed to validate the candidate gene, natural killer-tumor recognition sequence $(N K T R)$. The number of differentially expressed genes (DEGs) for stage IB, IIB, IIIA and IV tumors were 499, 602, 592 and 457, respectively. Most of the DEGs were NSCLC-related genes identified through literature research. A few genes were commonly downregulated in all the 4 stages of tumors, such as $C N T N 6$ and $L B X 2$. The DEGs in early-stage tumors were closely related with the negative regulation of signal transduction, the apoptosis pathway and the p53 signaling pathway. DEGs in
\end{abstract}

Correspondence to: Dr Shiying Zheng, Department of Cardiothoracic Surgery, The First Affiliated Hospital of Suzhou University, 188 Shizi Street, Suzhou, Jiangsu 215006, P.R. China

E-mail: shiyingzhengzhsy@163.com

*Contributed equally

Abbreviations: NSCLC, non-small cell lung cancer; PPI, protein-protein interaction; NCBI GEO, National Center for Biotechnology Information Gene Expression Omnibus; DAVID, Database for Annotation, Visualization and Integrated Discovery; GO, Gene Ontology; KEGG, Kyoto Encyclopedia of Genes and Genomes; CTD, Comparative Toxicogenomics Database

Key words: non-small cell lung cancer, stages, pathways, key nodes, coexpression gene pairs, tumor-related genes late-stage tumors were significantly enriched in transcription, response to organic substances and synapse regulation-related biological processes. A total of 16 genes (including NKTR) made up the significant coexpression network. NKTR was a key node in the PPI network and was significantly upregulated in lung cancer cells. The mechanism of NSCLC progression in different tumor stages may be different. NKTR may be the target candidate for NSCLC prevention and treatment.

\section{Introduction}

Non-small cell lung cancer (NSCLC) is the most common type of lung cancer, accounting for about $85 \%$ of all lung cancers (1). NSCLC is the main cause of lung cancer-related deaths. Most patients are diagnosed with late-stage tumors and only $15 \%$ of patients are found in the early stage (2). The survival rate of patients with early-stage NSCLC after complete tumor resection is more favourable. The current therapeutic strategies for NSCLC cases mainly include surgery, chemotherapy and radiotherapy. The combination of two or more treatments is often used for NSCLC cases, which is usually influenced by NSCLC staging. It is reported that the combinational treatment of adjuvant carboplatin plus paclitaxel reduces the mortality rate of patients with stage IB NSCLC (3). The overall survival for stage IB-II NSCLC cases was found to improve after vinorelbine plus cisplatin treatment (4).

Microarray technology is a novel tool to analyze the global gene expression and decipher biological pathways (5). Advances in microarray technology have facilitated the development of novel therapies and the understanding of potential mechanisms of diseases. Based on gene expression arrays, Lu et al found a group of genes related with lung cancer metastasis such as $A P C, C D H 8$ and $I L 8 R B$, which may be used for predicting the survival of patients with stage I NSCLC and preparing a treatment plan (6). Jiang et al employed tissue lung microarray technology and suggested that aldehyde dehydrogenase 1, a marker for lung tumor stem cells, may be a therapeutic target for lung cancer (7).

The gene expression array of GSE21933 was developed from Asian lung cancer patients. With the microarray data, Lo et al 
have established that ARHGAP19, FRAT2g, PAFAH1B1 and ZNF322A involved in Rho activity, Wnt signaling, motility control and MAPK signaling are the candidate genes for lung cancer (8). Currently, the integrity analysis of altered gene expression patterns for lung cancer during different stages is rare. In the present study, we further analyzed the expression array data of lung tumor tissues to disclose the difference and similarity in the gene expression profile of lung cancer cases during different stages. We expect that our findings are helpful in understanding the mechanism of lung cancer during different stages and discovering novel candidate genes.

\section{Materials and methods}

Microarray data. The gene expression dataset of a previous human NSCLC study was retrieved from the public National Center for Biotechnology Information Gene Expression Omnibus (NCBI GEO) with accession no. GSE21933. The gene expression data of tumors and matched normal tissues from 21 NSCLC patients were contributed by a previously public study (8). The NSCLC patients included 10 early-stage cases (6 in stage IB, 3 in stage IIB and 1 in stage IA) and 11 late-stage patients (5 in stage IIIA, 5 in stage IV and 1 in stage IIIB). Due to the small number of samples at stage IA and IIIB, we only analyzed the remaining 19 tumor samples compared with the corresponding controls. All the DNA microarray data were analyzed by the platform Phalanx Human OneArray.

Data preprocessing. The raw data (gpr format files) were downloaded and assessed by limma software $(9,10)$. The preprocessing procedure involved a background correction, gene expression normalization and microarray data condensing. Then, the probe IDs were translated into gene symbols by eliminating the probes with no gene symbols. When there were multiple probes for the same gene symbol, the mean value of the probes was calculated as the gene expression value.

$D E G$ screening. The differentially expressed genes (DEGs) were analyzed in NSCLC tumors during different stages. The differential expression patterns were analyzed in stages IB, IIB, IIIA and IV of lung tumors, compared with the normal controls. The significant P-values for DEGs were calculated by a no-paired t-test with the application of a limma package. $\mathrm{P}<0.05$ and $\log _{2} \mathrm{FC}$ (fold-change) $\geq 0.4$ were defined as the cut-off values. Then the significant DEGs screened were subjected to hierarchical clustering analysis by a gplots package (11).

Function and pathway analysis. The significantly altered biological processes and pathways were identified using Database for Annotation, Visualization and Integrated Discovery (DAVID) (12). The statistical significances of the overrepresented Gene Ontology (GO) terms and Kyoto Encyclopedia of Genes and Genomes (KEGG) pathways were calculated based on hypergeometric distribution. A P-value $<0.05$ and a count $\geq 2$ were used as the cut-off values.

PPI network. The protein interaction pairs corresponding to DEGs were predicted by integrating the information deposited in STRING [protein-protein interaction (PPI) networks, with increased coverage and integration] (13). The DEG lists in tumors in stages IB, IIB, IIIA and IV were analyzed, respectively. The PPI network was constructed of the protein pairs with a PPI score (medium confidence) $\geq 0.4$ using Cytoscape software (14).

Key nodes in the PPI network. The role of nodes in large complex networks is commonly assessed based on network centralities such as degree centrality (15), betweenness centrality (16) and subgraph centrality (17). The network centralities of nodes were calculated by cytoscape plugin CytoNCA (18). The key nodes were first selected according to a high degree, subgraph and betweenness value. Then the DEGs corresponding to key nodes underwent cluster analysis to evaluate the effect on sample clustering. Finally, DEGs that could distinguish tumor and normal samples were defined as key nodes.

Venn diagram of DEGs in tumors of different stages. VennPlex (19) (https://www.irp.nia.nih.gov/bioinformatics/vennplex.html) has been widely used to separate common DEGs and uniquely altered genes between different gene lists. The Venn diagram of DEGs in the NSCLC tumors of different stages was constructed by VennPlex software. The four DEG lists and $\log _{2} \mathrm{FC}$ values were uploaded to identify the difference of differential expression patterns among stages IB, IIB, IIIA and IV of lung tumors.

Gene coexpression analysis. The DEGs identified in the four different tumor stages were pooled together. The coexpression coefficient was calculated by Pearson's correlation coefficient. $\mathrm{P}$-values were obtained based on $\mathrm{Z}$-scores and corrected by Benjamini-Hochberg (BH) method to control the false discovery rate (20). A Icoexpression coefficientl $>0.8$ and an adjusted P-value $<0.05$ were set as the cut-off values for gene coexpression pairs.

Analysis of cancer-related genes. The Comparative Toxicogenomics Database (CTD) (21) is a collection of disease-related genes documented in previously published literature. The genes related with NSCLC were selected among DEGs of tumors in 4 tumor stages based on the information from the CTD.

Cell culture. Human lung epithelial cells (BEAS-2B), NSCLC cell lines such as A549 (adenocarcinoma), NCI-H1299 (large cell carcinoma) and NCI-H1650 (adenocarcinoma) were purchased from the Cell Bank of the Chinese Academy of Sciences (Shanghai, China). BEAS-2B and A549 cells were maintained in Dulbecco's modified Eagle's medium (DMEM) supplemented with $10 \%$ fetal bovine serum (FBS), $1 \%$ $100 \mathrm{U} / \mathrm{ml}$ penicillin and $0.1 \mathrm{mg} / \mathrm{ml}$ streptomycin. NCI-H1299 and NCI-H1650 cells were cultured in RPMI-1640 medium supplemented with $10 \% \mathrm{FBS}$ and $1 \% 100 \mathrm{U} / \mathrm{ml}$ penicillin, and $0.1 \mathrm{mg} / \mathrm{ml}$ streptomycin. All the cells were incubated in an atmosphere of $95 \%$ air and $5 \% \mathrm{CO}_{2}$ at $37^{\circ} \mathrm{C}$.

Reverse transcription real-time quantitative polymerase chain reaction $(R T-q P C R)$. The total RNA of the BEAS-2B, A549, NCI-H1299 and NCI-H1650 cells was extracted using 
Table I. The primer sequences for NKTR and GAPDH.

\begin{tabular}{ll}
\hline Gene & \multicolumn{1}{c}{ Primer sequence (5'-3') } \\
\hline GAPDH & F: TGACAACTTTGGTATCGTGGAAGG \\
& R: AGGCAGGGATGATGTTCTGGAGAG \\
NKTR & F: ACAGTTACCACCGAGGCAGA \\
& R: TCGGTCACTTTCACTGTCAGAGC
\end{tabular}

NKTR, natural killer-tumor recognition sequence; GAPDH, glyceraldehyde-3-phosphate dehydrogenase; $\mathrm{F}$, forward; $\mathrm{R}$, reverse.

the RNAiso Plus reagent (Takara Bio) according to the manufacturer's instructions. cDNA was reversely transcribed by PrimeScript (according to Takara Bio) at $37^{\circ} \mathrm{C}$ for $15 \mathrm{~min}$, followed by $5 \mathrm{sec}$ at $85^{\circ} \mathrm{C}$. In order to determine the DNA copy number variations of the candidate natural killer-tumor recognition sequence (NKTR) gene, PCR amplifications were performed with $10 \mu 1$ SYBR Premix Ex Taq (2X), $1 \mu 1$ of $10 \mu \mathrm{M}$ forward primers, $1 \mu \mathrm{l}$ of $10 \mu \mathrm{M}$ reverse primers and $8 \mu \mathrm{l}$ cDNA, in a final volume of $20 \mu \mathrm{l}$. The relative quantities of NKTR expression were analyzed using the comparative $\mathrm{Ct}$ method and were normalized to glyceraldehyde-3-phosphate dehydrogenase (GAPDH) expression. The primer sequences for NKTR and GAPDH are listed in Table I.

\section{Results}

DEG identification. Compared with normal tissues, the genes with altered expression in NSCLC tumors were identified (Table II). The total number of DEGs in tumor tissues of stage IB, IIB, IIIA and IV were 499, 602, 592 and 457, respectively. Then, the DEGs for different tumor stages were subjected to hierarchical clustering analysis based on the gene expression patterns. The heat maps illustrated that all the DEGs were able to distinguish tumor and normal tissues clearly, which suggested that the DEGs identified in the present study were significant (Fig. 1).

Significant GO function and pathways. The overrepresented GO terms and pathways for DEGs varied according to tumor stage. The DEGs in tumors of IB tumor stage were closely related with negative regulation of signal transduction and cellular di- and trivalent inorganic cation homeostasis biological process and the apoptosis pathway. The genes altered in stage IIB were significantly enriched in genitalia development, the monosaccharide metabolic process and the p53 signaling pathway. The upregulated DEGs in tumors of stages IIIA and IV were mainly related with transcription and synapse regulation-related biological process (Table III).

PPI network construction. The PPI network for DEGs in tumors of different stages was constructed respectively. The PPI network for stage IB was composed of 276 nodes and 454 pairs. The network for stage IIB included 349 nodes and 615 protein pairs. In addition, there were 366 nodes and 944 pairs for stage IIIA tumors and 252 nodes and 392 pairs for stage IV (Fig. 2).

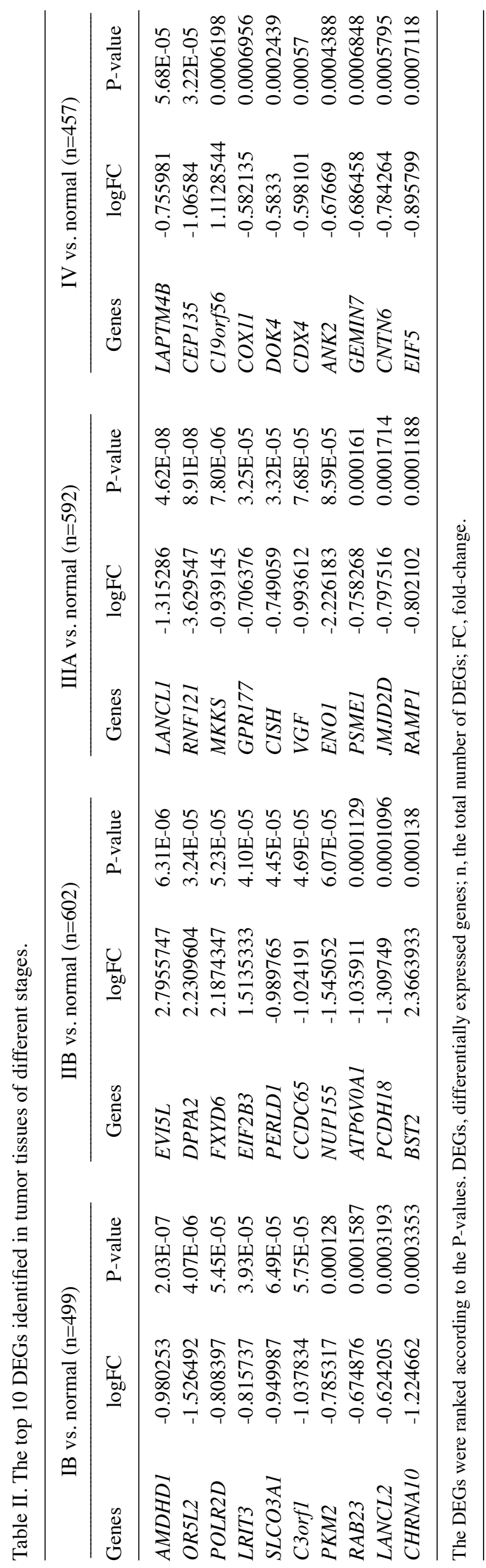



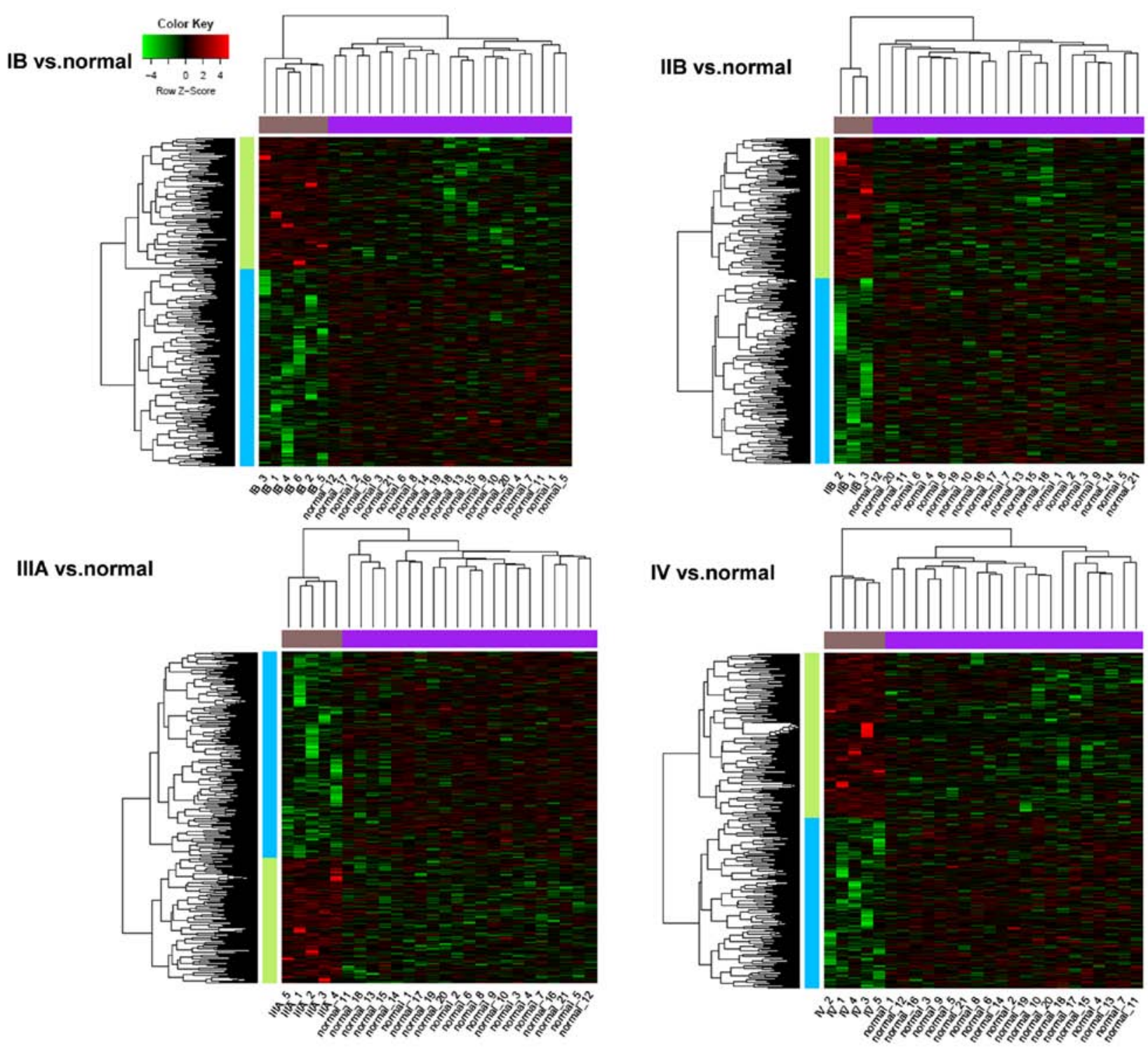

Figure 1. Hierarchical clustering analysis of DEGs in tumor of stages IB, IIB, IIIA and IV. Red, high expression level; green, low expression level. DEGs, differentially expressed genes.

Identification of the key nodes in the PPI network. Key nodes in the PPI network were identified by integration analysis of the scores calculated based on network centrality and the results of the clustering analysis. There were 29 key nodes in the stage IB group, 33 in the IIB group, 35 in the IIIA group and 54 in the IV group (Fig. 2). All the key nodes identified in the present study were differentially expressed and through their expression patterns we were able to distinguish normal and tumor tissues (Fig. 3). The key nodes were significantly enriched in cancer-related pathways, chronic myeloid leukemia, the chemokine signaling pathway, gap junctions and the insulin signaling pathway (Table IV).

Venn diagram of DEGs. As shown in Fig. 4, the number of genes that were differentially expressed at each tumor stage was assessed and the diagrams also indicated the common DEGs in tumors of stages IB, IIB, IIIA and IV. Most DEGs were uniquely identified in a single tumor stage and a few genes were commonly differentially expressed in the 4 tumor stages, such as downregulated CNTN6 and LBX2.
Identification of the coexpression gene pairs. A total of 1,984 genes were obtained by pooling the DEGs from different tumor stages, among which the coexpression gene pairs were analyzed. The coexpression network was constructed with 102 nodes and 179 gene interaction pairs (Fig. 5). Among the 102 nodes, 16 genes were involved in 65 gene pairs, such as SEMA4C, C19orf56, MYBBPIA, SPTA1, NKTR, WHSCIL1, FARP2, CDH18, C5orf33, ZNF124, ESRRG, ARHGEF10L, $R A B G A P 1, Z M Y M 1, P I P 5 K 1 B$ and PGBD3.

NSCLC-related gene identification. According to the CTD database, most of the genes were related with NSCLC during different stages. There were 372 genes $(74.55 \%)$ among all the DEGs in the IB stage group, 465 (77.24\%) for the IIB group, $466(78.72 \%)$ in the IIIA group and $353(77.24 \%)$ for the IV group (Table V).

mRNA expression of NKTR. RT-PCR analysis of NKTR in A549, NCI-H1299 and NCI-H1650 cells was conducted to establish the differential expression in NSCLC, compared 

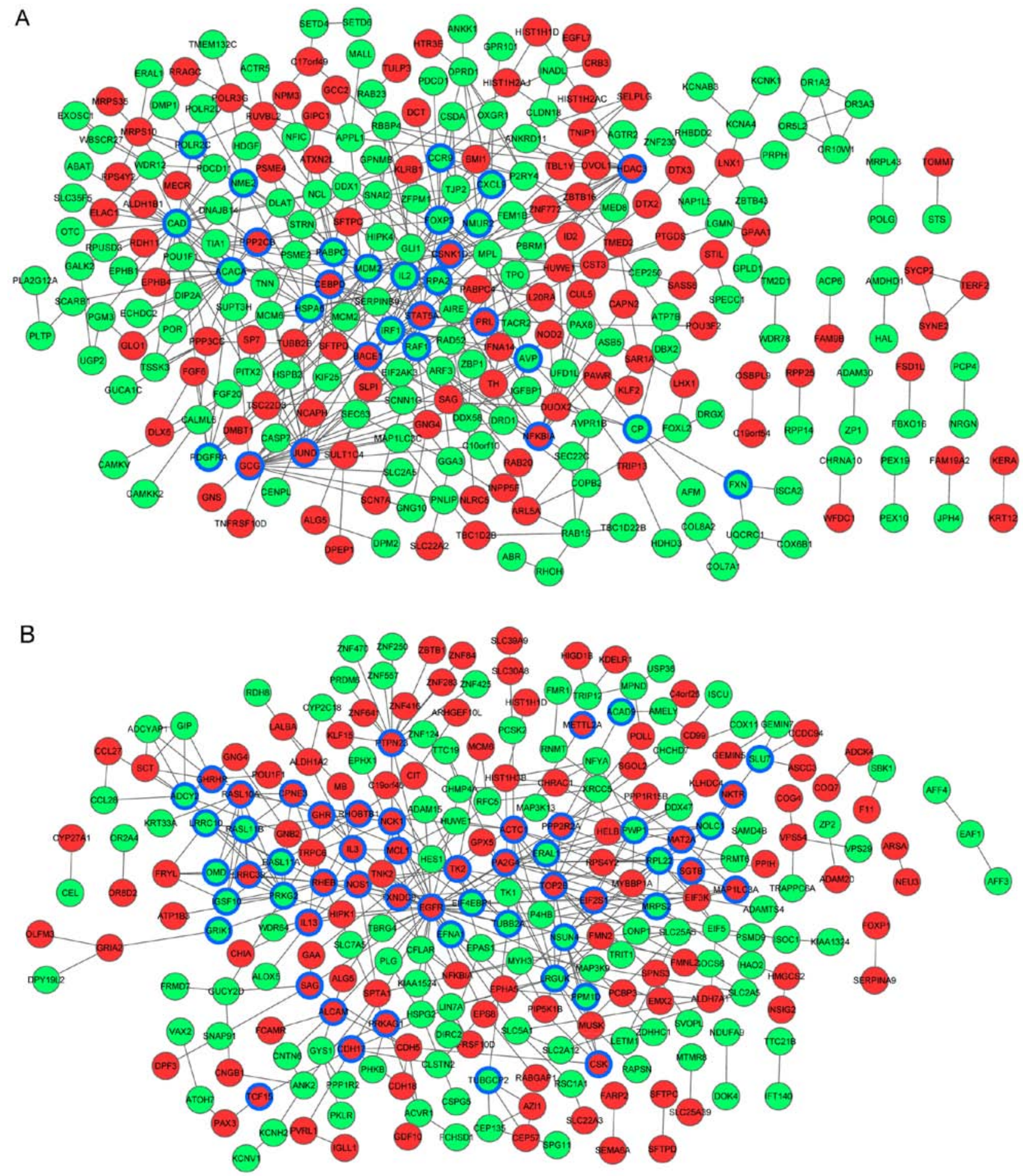

Figure 2. PPI network for DEGs in tumors of different stages. (A) PPI network for DEGs in tumors of stage IB. (B) PPI network for DEGs in tumors of stage IIB. Red, upregulated genes; green, downregulated genes; blue, key nodes. PPI, protein-protein interaction; DEGs, differentially expressed genes.

with the BEAS-2B cells. As shown in Fig. 6, the mean mRNA expression levels of NKTR in A549, NCI-H1299 and NCI-H1650 cells were significantly higher than those in the BEAS-2B cells $(\mathrm{P}<0.01)$, which corresponded to the DEG analysis and further confirmed its significance in NSCLC.

\section{Discussion}

In the present study, the gene expression profile analysis of NSCLC tissues of different stages revealed the significantly altered biological functions and pathways and proposed some candidate genes for lung cancer treatment.

The data (GSE21933) analyzed in the study were the cDNA microarray data. Both DNA and RNA microarray data can be used to analyze the expression levels of genes by limma software (1,2). The DEG analysis showed that there were 499, 602,592 and 457 genes differentially expressed in tumors of stages IB, IIB, IIIA and IV, respectively. Clustering analysis revealed that based on the expression level of DEGs, the tumor and normal samples were clearly distinguished. In addition, 

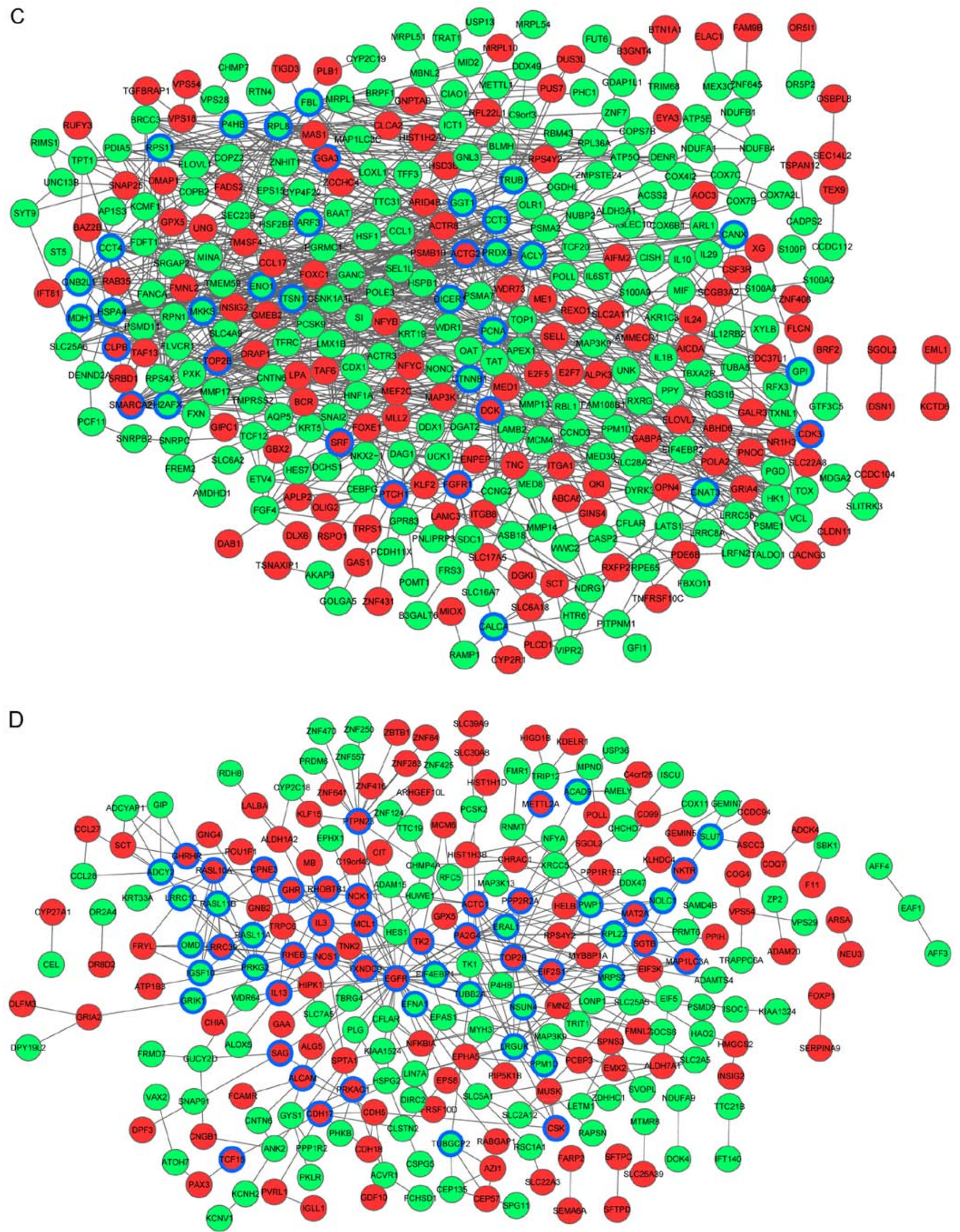

Figure 2. Continued. PPI network for DEGs in tumors of different stages. (C) PPI network for DEGs in tumors of stage IIIA. (D) PPI network for DEGs in tumors of stage IV. Red, upregulated genes; green, downregulated genes; blue, key nodes. PPI, protein-protein interaction; DEGs, differentially expressed genes.

literature research analysis confirmed that most of the DEGs identified in our study were NSCLC-related genes.These find- ings suggested that the DEGs established in our study were significant. 
Table III. The overrepresented GO terms and pathways for the DEGs.

\begin{tabular}{|c|c|c|c|c|c|}
\hline Stages & Regulated & Category & Term & Count & P-value \\
\hline \multirow[t]{7}{*}{ IB } & \multirow[t]{4}{*}{ Upregulated } & \multirow[t]{3}{*}{$\mathrm{BP}$} & GO:0009968 negative regulation of signal transduction & 9 & $1.25 \mathrm{E}-03$ \\
\hline & & & GO:0010648 negative regulation of cell communication & 9 & $2.58 \mathrm{E}-03$ \\
\hline & & & GO:0007128 meiotic prophase I & 3 & $4.69 \mathrm{E}-03$ \\
\hline & & Pathway & hsa04210:Apoptosis & 4 & 4.57E-02 \\
\hline & \multirow[t]{3}{*}{ Downregulated } & \multirow[t]{3}{*}{$\mathrm{BP}$} & GO:0030005 cellular di- and trivalent inorganic cation homeostasis & 12 & $6.93 \mathrm{E}-04$ \\
\hline & & & GO:0055066 di- and trivalent inorganic cation homeostasis & 12 & $1.05 \mathrm{E}-03$ \\
\hline & & & GO:0007204 elevation of cytosolic calcium ion concentration & 8 & $1.41 \mathrm{E}-03$ \\
\hline \multirow[t]{6}{*}{ IIB } & \multirow[t]{3}{*}{ Upregulated } & \multirow[t]{2}{*}{$\mathrm{BP}$} & GO:0048806 genitalia development & 3 & $3.52 \mathrm{E}-02$ \\
\hline & & & GO:0051054 positive regulation of the DNA metabolic process & 4 & $3.58 \mathrm{E}-02$ \\
\hline & & Pathway & hsa04115:p53 signaling pathway & 4 & $4.75 \mathrm{E}-02$ \\
\hline & \multirow[t]{3}{*}{ Downregulated } & \multirow[t]{3}{*}{$\mathrm{BP}$} & GO:0005996 monosaccharide metabolic process & 11 & $7.91 \mathrm{E}-03$ \\
\hline & & & GO:0019318 hexose metabolic process & 10 & 8.99E-03 \\
\hline & & & GO:0006006 glucose metabolic process & 8 & $2.27 \mathrm{E}-02$ \\
\hline \multirow[t]{6}{*}{ IIIA } & \multirow[t]{3}{*}{ Upregulated } & \multirow[t]{3}{*}{$\mathrm{BP}$} & $\begin{array}{l}\text { GO:0010552 positive regulation of specific transcription from } \\
\text { RNA polymerase II promoter }\end{array}$ & 5 & $4.89 \mathrm{E}-03$ \\
\hline & & & GO:0006350 transcription & 39 & $5.68 \mathrm{E}-03$ \\
\hline & & & GO:0016477 cell migration & 10 & $6.27 \mathrm{E}-03$ \\
\hline & \multirow[t]{3}{*}{ Downregulated } & \multirow[t]{3}{*}{$\mathrm{BP}$} & GO:0019320 hexose catabolic process & 8 & $3.74 \mathrm{E}-04$ \\
\hline & & & GO:0010033 response to organic substances & 29 & $3.78 \mathrm{E}-04$ \\
\hline & & & GO:0006091 generation of precursor metabolites and energy & 17 & $4.20 \mathrm{E}-04$ \\
\hline \multirow[t]{6}{*}{ IV } & \multirow[t]{3}{*}{ Upregulated } & \multirow[t]{3}{*}{$\mathrm{BP}$} & GO:0050807 regulation of synapse organization & 4 & $1.81 \mathrm{E}-03$ \\
\hline & & & GO:0050803 regulation of synapse structure and activity & 4 & $2.69 \mathrm{E}-03$ \\
\hline & & & GO:0010033 response to organic substances & 18 & $4.82 \mathrm{E}-03$ \\
\hline & \multirow[t]{3}{*}{ Downregulated } & \multirow[t]{3}{*}{ BP } & GO:0050892 intestinal absorption & 3 & $1.41 \mathrm{E}-02$ \\
\hline & & & GO:0035036 sperm-egg recognition & 3 & $1.58 \mathrm{E}-02$ \\
\hline & & & GO:0007339 binding of sperm to zona pellucida & 3 & $1.58 \mathrm{E}-02$ \\
\hline
\end{tabular}

DEGs, differentially expressed genes; GO, Gene Ontology; BP, biological process.

Function analysis showed that the significantly altered biological process in the early lung cancer stage mainly included negative regulation of signal transduction, apoptosis, cellular di- and trivalent inorganic cation homeostasis (stage IB) and positive regulation of the DNA metabolic process and the p53 signaling pathway (stage IIB). Previous evidence has shown that lung cancer-related candidate genes are mainly related with apoptosis, signal transduction and DNA repair. The ion transport is a significantly altered biological process for both the Asian and Caucasian population, which may play a key role in tumorigenesis (8). p53 gene is a tumor-suppressor gene and is reported to be frequently mutated in lung cancer patients (22). Non-disruptive p53 mutations may decrease the survival rate in patients with advanced NSCLC (23). Signal transduction induced by focal adhesion kinase is found to inhibit the p53-mediated apoptosis in malignant cells (24).

In the late-stage NSCLC tumors, the DEGs were closely related with cell migration, response to organic substances (stage IIIA) and regulation of synapse organization, structure and activity (stage IV). Cell migration has been reported to be one of the significantly altered biological processes in NSCLC cancer patients (8), which is consistent with our findings. The biological process of response to organic substances was significantly altered in stage IIIA and IV NSCLC tumors, indicating that this biological process may play a dominant role in the late stages of lung cancer. The role of the synapse regulationrelated biological process has not been clarified in lung cancer progression. Thus, the altered biological processes warrant further study. Above all, the biological processes and pathways in NSCLC progression may be altered in a stage-dependent manner. The DEG enriched biological processes and pathways were different in early-stage and late-stage NSCLC, which was determined by Venn diagram analysis. The Venn diagram illustrated that a few genes had similar expression patterns in NSCLC at different stages, which revealed that NSCLC at different stages induced differential gene expression patterns leading to the dysfunction of various biological processes.

Additionally, after removing the overlapped genes, a total of 1,984 DEGs were obtained in tumors of stages IB, IIB, IIIA and IV. The coexpression network composed of 16 genes and 65 coexpression pairs was the most significant. The protein interaction pairs corresponding to DEGs can be predicted 


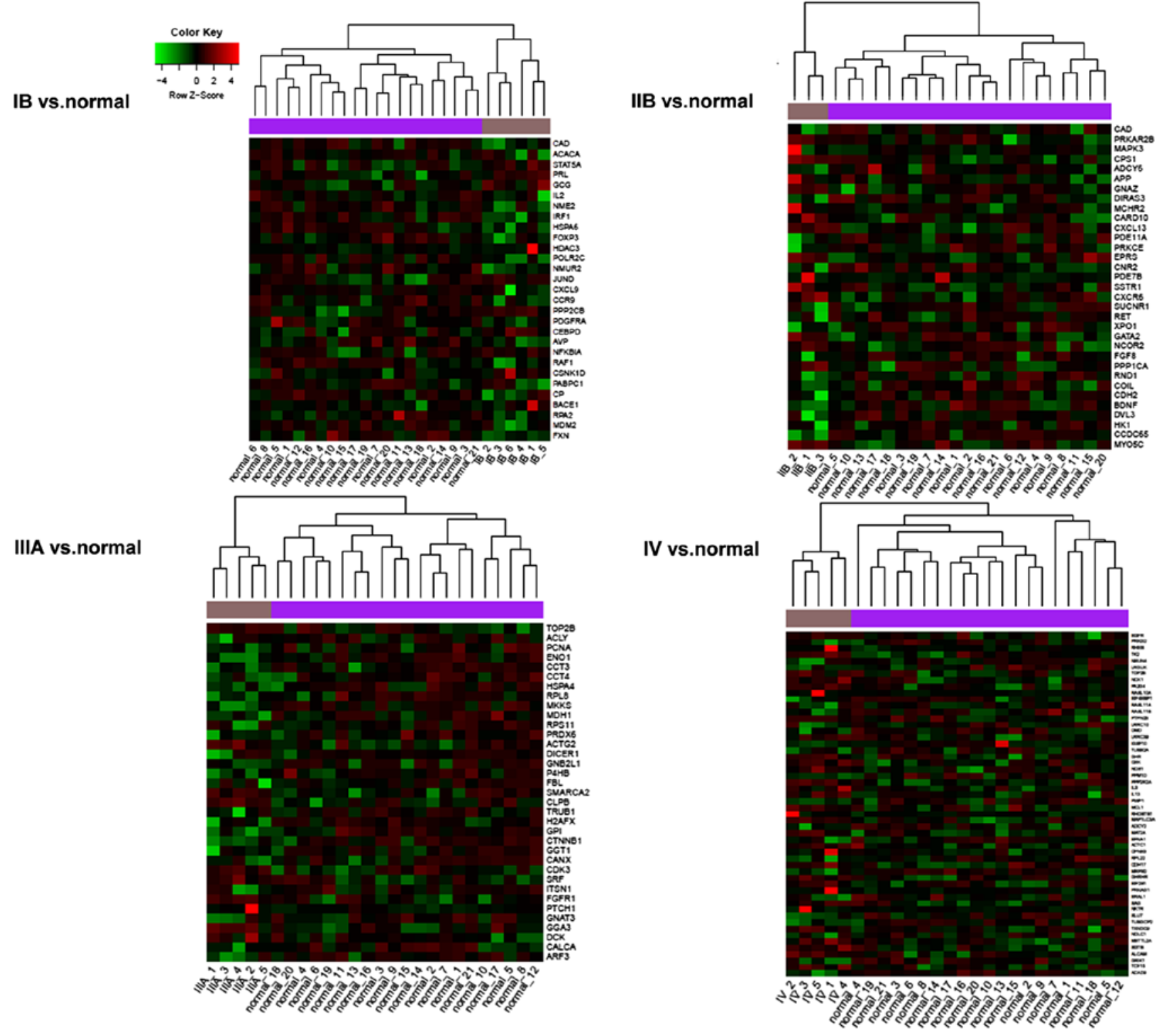

Figure 3. Hierarchical clustering analysis of the key nodes in the PPI network. PPI, protein-protein interaction.

Table IV. The significant pathways for key nodes in the PPI network.

\begin{tabular}{|c|c|c|c|}
\hline Stages & Term & P-value & Genes \\
\hline \multirow[t]{8}{*}{ IB } & hsa05220:Chronic myeloid leukemia & 2.93E-03 & STAT5A, NFKBIA, RAF1, MDM2 \\
\hline & hsa05215:Prostate cancer & 4.76E-03 & PDGFRA, NFKBIA, RAF1, MDM2 \\
\hline & hsa04060:Cytokine-cytokine receptor interaction & $1.74 \mathrm{E}-02$ & CCR9, PDGFRA, CXCL9, PRL, IL2 \\
\hline & hsa05214:Glioma & 2.49E-02 & PDGFRA, RAF1, MDM2 \\
\hline & hsa05218:Melanoma & $3.11 \mathrm{E}-02$ & PDGFRA, RAF1, MDM2 \\
\hline & hsa04062:Chemokine signaling pathway & $3.52 \mathrm{E}-02$ & CCR9, CXCL9, NFKBIA, RAF1 \\
\hline & hsa05200:Pathways in cancer & $3.62 \mathrm{E}-02$ & STAT5A, PDGFRA, NFKBIA, RAF1, MDM2 \\
\hline & hsa04540:Gap junction & 4.69E-02 & CSNK1D, PDGFRA, RAF1 \\
\hline \multirow[t]{3}{*}{ IIB } & hsa04270:Vascular smooth muscle contraction & $1.50 \mathrm{E}-02$ & PPPICA, MAPK3, ADCY6, PRKCE \\
\hline & hsa04930:Type II diabetes mellitus & 2.03E-02 & $M A P K 3, H K 1, P R K C E$ \\
\hline & hsa04910:Insulin signaling pathway & $2.46 \mathrm{E}-02$ & $P R K A R 2 B, P P P 1 C A, M A P K 3, H K 1$ \\
\hline IV & hsa04540:Gap junction & 7.13E-03 & $E G F R, A D C Y 2, T U B B 2 A, P R K G 2$ \\
\hline
\end{tabular}

PPI, protein-protein interaction. 


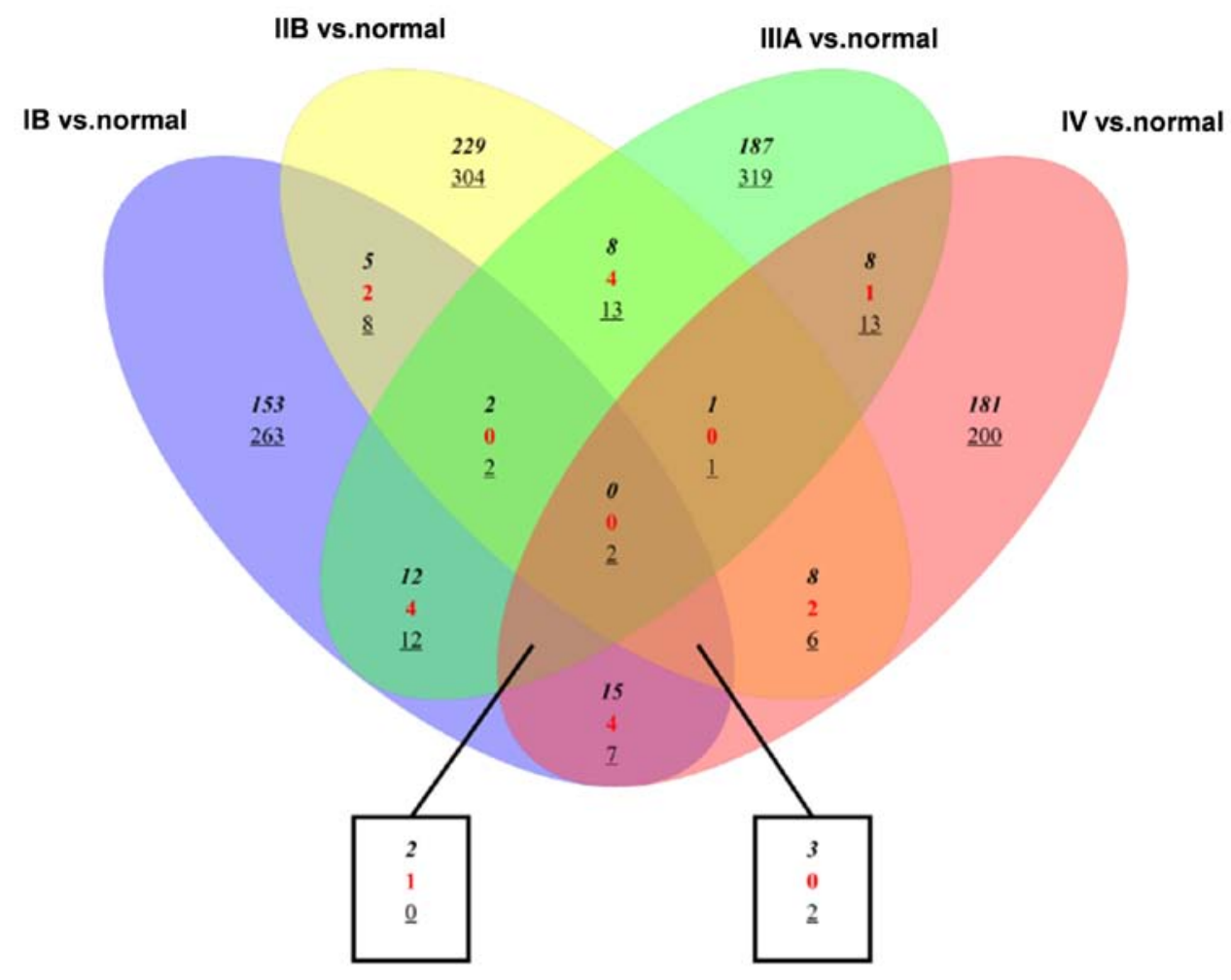

Figure 4. Venn diagram for DEGs in tumors of different stages. DEGs, differentially expressed genes.

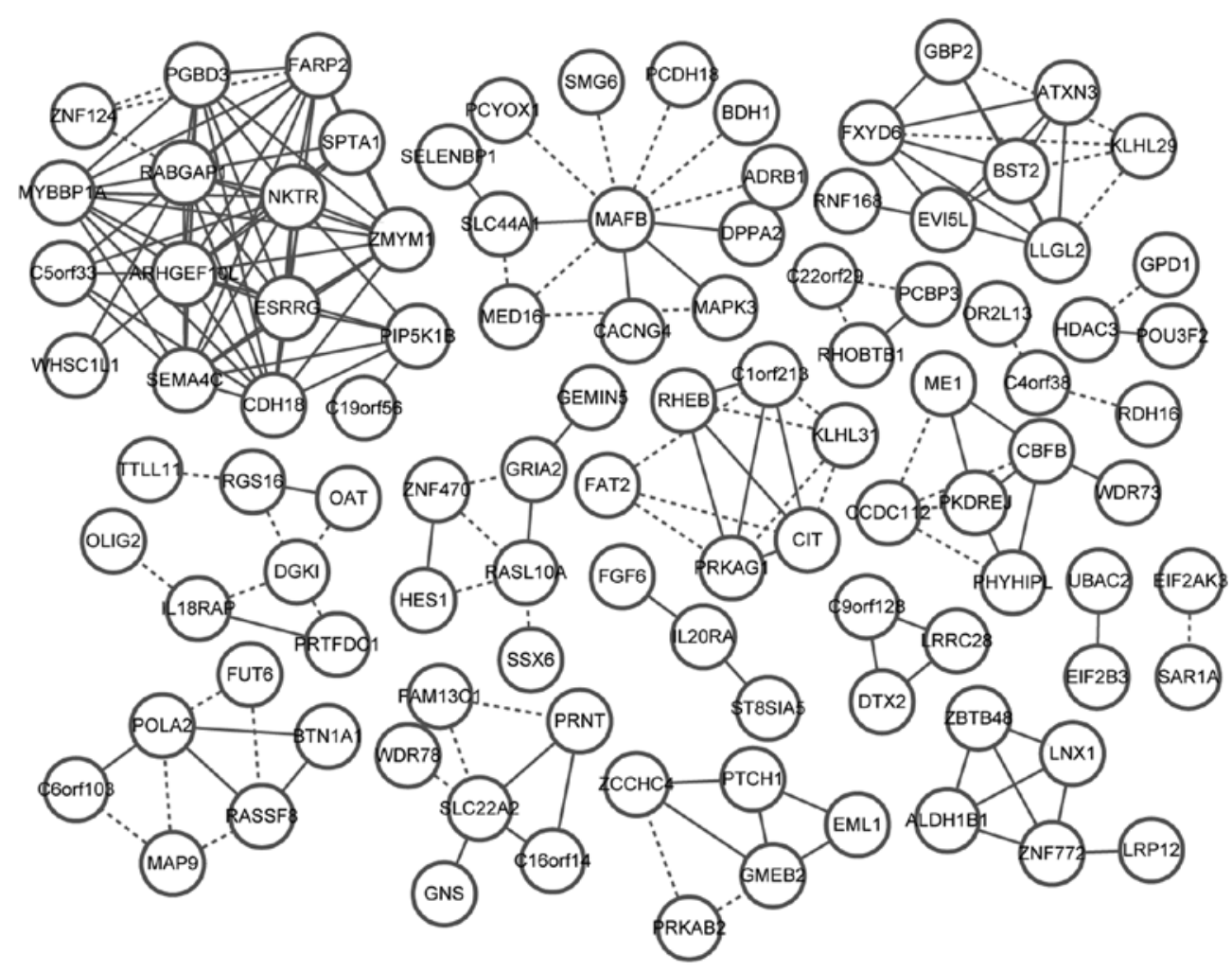

Figure 5. Coexpression gene network for DEGs in tumors of different stages. Full line, positive correlation; dashed line, negative correlation. DEGs, differentially expressed genes.

by integrating the information deposited in STRING (13). Cytoscape is a free software package and widely used in analyzing the results of mRNA expression profiling, functional genomic and proteomic experiments in the context of an interaction network obtained for genes of interest (14). Thus, protein interactions were able to be obtained based on the microarray 
Table V. NSCLC-related genes in tumors of different stages.

\begin{tabular}{lccc}
\hline Groups & $\begin{array}{c}\text { Total DEG } \\
\text { count }\end{array}$ & $\begin{array}{c}\text { Disease related } \\
\text { gene count }\end{array}$ & Percentage \\
\hline IB & 499 & 372 & 74.55 \\
IIB & 602 & 465 & 77.24 \\
IIIA & 592 & 466 & 78.72 \\
IV & 457 & 353 & 77.24 \\
\hline
\end{tabular}

NSCLC, non-small cell lung cancer; DEGs, differentially expressed genes.

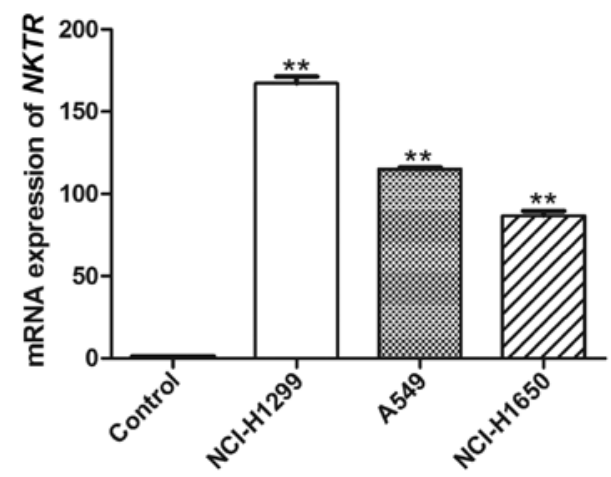

Figure 6. RT-PCR validation of the relative expression of NKTR in NSCLC cell lines. ${ }^{* * *} \mathrm{P}<0.01$, compared with the control.

data in the study. NKTR was one of the genes among the 16 genes that showed multiple coexpression interactions with others. Simultaneously, PPI analysis showed that NKTR was a key node in stage IV tumor tissues according to degree, betweenness and subgraph centrality. In addition, according to expression patterns of NKTR, tumor and normal tissues could be clearly distinguished. RT-PCR of gene validation analysis showed that NKTR was significantly upregulated in NSCLC cells, compared with normal epithelial cells.

All these findings indicated that NKTR is a significant DEG that plays a critical role in NSCLC progression. NKTR has been found to be differentially expressed in human lung squamous cell carcinoma and is located in chromosome 3p23-p21 according to the cDNA library (25). NKTR as a component of a spliceosome, has functions in protein folding. NKTR-102 is considered as a novel PEGylated-irinotecan conjugate, which functions in the inhibition of the growth of human colorectal and lung tumors (26). Although the role of NKTR in NSCLC has not been clarified, NKTR and its coexpressed genes may play a key role in NSCLC progression.

In this study, the microarray data of GSE21933 were developed from NSCLC patients including 10 early-stage patients (6 stage IB, 3 stage IIB and 1 stage IA) and 11 late-stage patients (5 stage IIIA, 5 stage IV and 1 stage IIIB). Due to the small number of samples at stage IA and IIIB, we only analyzed the remaining 19 tumor samples compared with the corresponding controls. In the original study, the microarray data of GSE21276 were produced from tumor and normal tissues of 40 Asian and 20 Caucasian NSLCL patients and was aimed to characterize the gene expression profiles in different racial groups. However, in the present study, we attempted to analyze the changes in gene expression profiles during the different tumor stages. The detailed information of the 19 patients was not provided in the original study, thus we cannot provide information on the type of tumors. In our present study, we performed gene validation experiments in A549 (adenocarcinoma), NCI-H1299 (large-cell carcinoma) and NCI-H1650 (adenocarcinoma) cells, and determined that NKTR was a candidate gene in NSLCL prevention and treatment.

Despite some previous microarray data that were also developed from a small number of samples $(27,28)$, the small sample size used may be a limitation in our study. Further studies with a larger size of samples are warranted in the near future.

In conclusion, the gene expression patterns are different at various stages of NSCLC tumors, from early-stage to late-stage, which lead to the dysfunction of different biological processes. Different mechanisms may be involved in the progression of NSCLC tumors from early-stage to late-stage. An adequate understanding of the pathogenesis underlying different tumor stages may be helpful in new therapy development. NKTR is commonly differentially expressed in stage IB, IIB, IIIA and IV tumors. NKTR and its coexpressed gene pairs may be candidate genes for the prevention and treatment of NSCLC.

\section{References}

1. Paez JG, Jänne PA, Lee JC, Tracy S, Greulich H, Gabriel S, Herman P, Kaye FJ, Lindeman N, Boggon TJ, et al: EGFR mutations in lung cancer: Correlation with clinical response to gefitinib therapy. Science 304: 1497-1500, 2004.

2. Edwards BK, Noone AM, Mariotto AB, Simard EP, Boscoe FP, Henley SJ, Jemal A, Cho H, Anderson RN, Kohler BA, et al: Annual Report to the Nation on the status of cancer, 1975-2010, featuring prevalence of comorbidity and impact on survival among persons with lung, colorectal, breast, or prostate cancer. Cancer 120: 1290-1314, 2014.

3. Strauss GMHJ, Maddaus MA, Johnstone DW, Johnson EA, Watson DM, Sugarbaker DJ, Schilsky RA, Vokes EE and Green MR: Adjuvant chemotherapy in stage IB non-small cell lung cancer (NSCLC): Update of Cancer and Leukemia Group B (CALGB) protocol 9633. In: ASCO Annual Meeting Abstract 24: Suppl 2007, 2006.

4. Winton T, Livingston R, Johnson D, Rigas J, Johnston M, Butts C, Cormier Y, Goss G, Inculet R, Vallieres E, et al; National Cancer Institute of Canada Clinical Trials Group; National Cancer Institute of the United States Intergroup JBR.10 Trial Investigators: Vinorelbine plus cisplatin vs. observation in resected non-small-cell lung cancer. N Engl J Med 352: 2589-2597, 2005

5. Huang Q, Dunn RT II, Jayadev S, DiSorbo O, Pack FD, Farr SB, Stoll RE and Blanchard KT: Assessment of cisplatin-induced nephrotoxicity by microarray technology. Toxicol Sci 63: 196-207, 2001.

6. Lu Y, Lemon W, Liu P-Y, Yi Y, Morrison C, Yang P, Sun Z, Szoke J, Gerald WL, Watson M, et al: A gene expression signature predicts survival of patients with stage I non-small cell lung cancer. PLoS Med 3: e467, 2006.

7. Jiang F, Qiu Q, Khanna A, Todd NW, Deepak J, Xing L, Wang H, Liu Z, Su Y, Stass SA, et al: Aldehyde dehydrogenase 1 is a tumor stem cell-associated marker in lung cancer. Mol Cancer Res 7: 330-338, 2009.

8. Lo FY, Chang JW, Chang IS, Chen YJ, Hsu HS, Huang SF, Tsai FY, Jiang SS, Kanteti R, Nandi S, et al: The database of chromosome imbalance regions and genes resided in lung cancer from Asian and Caucasian identified by array-comparative genomic hybridization. BMC Cancer 12: 235, 2012.

9. Ritchie ME, Phipson B, Wu D, Hu Y, Law CW, Shi W and Smyth GK: limma powers differential expression analyses for RNA-sequencing and microarray studies. Nucleic Acids Res 43: e47, 2015 . 
10. Smyth GK: limma: linear models for microarray data. In Bioinformatics and Computational Biology Solutions Using R and Bioconductor. Gentleman R, Carey VJ, Huber W, Irizarry RA and Dudoit S (eds). Springer, NY, New York, pp397-420, 2005.

11. Warnes GR, Bolker B, Bonebakker L, Gentleman R, Liaw WHA, Lumley T, Maechler M, Magnusson A, Moeller S, Schwartz M, et al: gplots: Various R programming tools for plotting data. R package version 2, 2009. https://cran.r-project.org/web/packages/ gplots.

12. Huang W, Sherman BT and Lempicki RA: Systematic and integrative analysis of large gene lists using DAVID bioinformatics resources. Nat Protoc 4: 44-57, 2009.

13. Szklarczyk D, Franceschini A, Wyder S, Forslund K, Heller D, Huerta-Cepas J, Simonovic M, Roth A, Santos A, Tsafou KP, et al: STRING v10: protein-protein interaction networks, integrated over the tree of life. Nucleic Acids Res 43: D447-D452, 2015.

14. Shannon P, Markiel A, Ozier O, Baliga NS, Wang JT, Ramage D, Amin N, Schwikowski B and Ideker T: Cytoscape: A software environment for integrated models of biomolecular interaction networks. Genome Res 13: 2498-2504, 2003.

15. Opsahl T, Agneessens F and Skvoretz J: Node centrality in weighted networks: Generalizing degree and shortest paths. Soc Networks 32: 245-251, 2010.

16. Goh KI, Oh E, Kahng B and Kim D: Betweenness centrality correlation in social networks. Phys Rev E Stat Nonlin Soft Matter Phys 67: 017101, 2003.

17. Estrada E and Rodríguez-Velázquez JA: Subgraph centrality in complex networks. Phys Rev E Stat Nonlin Soft Matter Phys 71: 056103, 2005.

18. Tang Y, Li M, Wang J, Pan Y and Wu FX: CytoNCA: A cytoscape plugin for centrality analysis and evaluation of protein interaction networks. Biosystems 127: 67-72, 2015.

19. Cai H, Chen H, Yi T, Daimon CM, Boyle JP, Peers C, Maudsley S and Martin B: VennPlex - a novel Venn diagram program for comparing and visualizing datasets with differentially regulated datapoints. PLoS One 8: e53388, 2013.

20. Liang M, Zhang F, Jin G and Zhu J: FastGCN: A GPU accelerated tool for fast gene co-expression networks. PLoS One 10: e0116776, 2015 .
21. Davis AP, Grondin CJ, Lennon-Hopkins K, Saraceni-Richards C, Sciaky D, King BL, Wiegers TC and Mattingly CJ: The Comparative Toxicogenomics Database's 10th year anniversary: Update 2015. Nucleic Acids Res 43: D914-D920, 2015.

22. Gibbons DL, Byers LA and Kurie JM: Smoking, p53 mutation, and lung cancer. Mol Cancer Res 12: 3-13, 2014.

23. Molina-Vila MA, Bertran-Alamillo J, Gascó A, Mayo-delas-Casas C, Sánchez-Ronco M, Pujantell-Pastor L, Bonanno L, Favaretto AG, Cardona AF, Vergnenègre A, et al: Nondisruptive p53 mutations are associated with shorter survival in patients with advanced non-small cell lung cancer. Clin Cancer Res 20: 4647-4659, 2014.

24. Ilić D, Almeida EA, Schlaepfer DD, Dazin P, Aizawa S and Damsky $\mathrm{CH}$ : Extracellular matrix survival signals transduced by focal adhesion kinase suppress p53-mediated apoptosis. J Cell Biol 143: 547-560, 1998.

25. Sun W, Zhang K, Zhang X, Lei W, Xiao T, Ma J, Guo S, Shao S, Zhang H, Liu Y, et al: Identification of differentially expressed genes in human lung squamous cell carcinoma using suppression subtractive hybridization. Cancer Lett 212: 83-93, 2004.

26. Eldon MA, Staschen CM, Viegas T and Bentley M: NKTR-102, a novel PEGylated-irinotecan conjugate, results in sustained tumor growth inhibition in mouse models of human colorectal and lung tumors that is associated with increased and sustained tumor SN38 exposure. In: Aacr-Nci-Eortc International Conference on Molecular Targets and Cancer Therapeutics, San Franciso, 2007.

27. Ramadori G, Konstantinidou G, Venkateswaran N, Biscotti T, Morlock L, Galié M, Williams NS, Luchetti M, Santinelli A, Scaglioni PP and Coppari R: Diet-induced unresolved ER stress hinders KRAS-driven lung tumorigenesis. Cell Metab 21: 117-125, 2015.

28. Stueckle TA, Lu Y, Davis ME, Wang L, Jiang BH, Holaskova I, Schafer R, Barnett JB and Rojanasakul Y: Chronic occupational exposure to arsenic induces carcinogenic gene signaling networks and neoplastic transformation in human lung epithelial cells. Toxicol Appl Pharmacol 261: 204-216, 2012. 